\title{
Religiosity and Patient Activation Among Hospital Survivors of an Acute Coronary Syndrome
}

\author{
Hawa O. Abu, MD, MPH, PhD ${ }^{\top}$ (D) David D. McManus, MD, MSCF, \\ Catarina I. Kiefe, $P h D, M D^{7}$, and Robert J. Goldberg, $P h D^{7}$ \\ 'Department of Population and Quantitative Health Sciences, University of Massachusetts Medical School, Worcester, MA, USA; ${ }^{2}$ Division of \\ Cardiovascular Medicine, Department of Medicine, University of Massachusetts Medical School, Worcester, MA, USA.
}

BACKGROUND: Optimum management after an acute coronary syndrome (ACS) requires considerable patient engagement/activation. Religious practices permeate people's lives and may influence engagement in their healthcare. Little is known about the relationship between religiosity and patient activation.

OBJECTIVE: To examine the association between religiosity and patient activation in hospital survivors of an ACS.

DESIGN: Secondary analysis using baseline data from Transitions, Risks, and Actions in Coronary Events: Center for Outcomes Research and Education (TRACECORE) Study.

PARTICIPANTS: A total of 2067 patients hospitalized for an ACS at six medical centers in Central Massachusetts and Georgia (2011-2013).

MAIN MEASURES: Study participants self-reported three items assessing religiosity - strength and comfort from religion, making petition prayers, and awareness of intercessory prayers for health. Patient activation was assessed using the 6-item Patient Activation Measure (PAM-6). Participants were categorized as either having low (levels 1 and 2) or high (levels 3 and 4) activation.

RESULTS: The mean age of study participants was 61 years, 33\% were women, and $81 \%$ were non-Hispanic White. Approximately 85\% derived strength and comfort from religion, $61 \%$ prayed for their health, and $89 \%$ received intercessory prayers for their health. Overall, 58\% had low activation. Reports of a great deal (aOR, 2.02; 95\% CI, 1.44-2.84), and little/some (aOR, 1.45; 95\% CI, 1.07-1.98) strength and comfort from religion were associated with high activation, as were receipt of intercessions (aOR, 1.48; 95\% CI, 1.07-2.05). Praying for one's health was associated with low activation (aOR, 0.78; 95\% CI, 0.61-0.99).

Prior Presentation Abu HO, McManus DD, Kiefe CI, Goldberg RJ. Religiosity and Patient Engagement in their Healthcare Among Hospital Survivors of an Acute Coronary Syndrome. Abstract Poster Presented at the University of Massachusetts Community Engagement and Research Symposium March 22, 2019. Worcester MA, USA.

Electronic supplementary material The online version of this article (https://doi.org/10.1007/s11606-019-05345-4) contains supplementary material, which is available to authorized users.

Received April 17, 2019

Accepted September 6, 2019

Published online November 1, 2019
CONCLUSIONS: Most ACS survivors acknowledge religious practices toward their recovery. Strength and comfort from religion and intercessory prayers for health were associated with high patient activation. Petition prayers for health were associated with low activation. Healthcare providers should use knowledge about patient's religiosity to enhance patient engagement in their care.

KEY WORDS: patient activation; patient engagement; self-management; complementary and integrative medicine; religiosity; spirituality; acute coronary syndrome.

J Gen Intern Med 35(3):762-9

DOI: $10.1007 / \mathrm{s} 11606-019-05345-4$

(c) Society of General Internal Medicine 2019

\section{INTRODUCTION}

Approximately 1.1 million American adults are hospitalized annually for an acute coronary syndrome (ACS). ${ }^{1}$ The American Heart Association (AHA) recommends that patients with ACS become actively engaged in their management by undertaking lifestyle modifications and adhere to prescribed medications. ${ }^{2}$ For sustained and effective self-management, ACS survivors require the appropriate skills, beliefs, and motivation to implement recommended lifestyle changes.

Patient activation describes patients' understanding of their role in their healthcare plan and the extent of selfmanagement. ${ }^{3}$ Higher levels of patient activation have been associated with improved health outcomes and greater patient satisfaction. ${ }^{4}$ Assessing patient activation in the period following a life-threatening illness provides an opportunity for providers to tailor their care plans to meet patient needs prior to hospital discharge. ${ }^{5}$

When faced with life-threatening conditions, such as ACS, patients may experience existential concerns, and turn to their religious beliefs or faith to cope with their illness. ${ }^{6-8}$ Religious practices may facilitate the adoption of healthier lifestyle behaviors and are important in shaping how people perceive their health and engage in selfcare. ${ }^{9,10}$ Certain religious beliefs may, however, conflict with recommendations by medical professionals, inhibit healthcare utilization, and may lead to poor treatment adherence. ${ }^{11}$ At one extreme, patients who exhibit a passive dimension of "spiritual health locus of control" (sense of 
lack of control over health and illness with the belief that health outcomes are predetermined by a higher power) may not take an active role in their healthcare. ${ }^{12}$ In contrast, those with an active "spiritual health locus of control" may be better motivated to manage their illness. ${ }^{12}$

Religious beliefs and practices are common among patients seeking medical care. ${ }^{13}$ Therefore, a better understanding of how religiosity may facilitate or hinder patient engagement in their healthcare provides a more holistic approach in patientcentered care. We examined the association between religiosity and patient activation in a large patient cohort hospitalized for ACS.

\section{METHODS}

\section{Study Population}

This investigation used baseline data from the Transitions, Risks and Actions in Coronary Events: Center for Outcomes Research and Education (TRACE-CORE) Study. ${ }^{14,15}$ In brief, TRACE-CORE used a multi-center prospective design to recruit 2174 patients hospitalized with ACS at three tertiary care and community medical centers in Worcester, MA, two tertiary care hospitals in Atlanta, GA, and a large teaching hospital in Macon, GA, between April 2011 and May 2013. Eligible participants were aged $\geq 21$ years and discharged from the participating medical centers following index hospitalization for ACS. The Institutional Review Boards at participating sites approved this study. Written informed consent was obtained from each participant.

Trained interviewers conducted a computer-assisted inperson baseline interview during the index hospitalization or by telephone within $72 \mathrm{~h}$ of discharge, and abstracted detailed information from hospital medical records at study enrollment. Each validated case of ACS was categorized as either unstable angina, non-ST segment elevation myocardial infarction (NSTEMI), or ST segment elevation myocardial infarction (STEMI) based on standard criteria ${ }^{16}$; indeterminate ACS cases were adjudicated by a team of study physicians masked to the patient's diagnosis.

\section{Assessment of Religiosity}

During hospitalization, patients self-reported three items assessing religiosity. The first item asked: "How much is religion a source of strength and comfort to you?" with response options "none", "a little", "some", and "a great deal". Responses of "a little" or "some" were combined for analysis since the groups were similar and had too few outcomes to be examined separately. The second item assessed petition prayers for health by asking, "Do you use prayer specifically for your health?" The third item assessed intercessory prayers for health by asking "Do you know of others outside of your family who are praying for your health?" Responses to the second and third item included "Yes" or "No".

\section{Patient Activation}

We examined patient activation during hospitalization with an abbreviated 6-item version of the validated 13-item Patient Activation Measure (PAM) (Table 1). The PAM assessed patient's knowledge, skills, and confidence in self-care. ${ }^{17}$ Participant responses were assessed on a 4-point Likert scale ranging from "strongly disagree" to "strongly agree." Based on the developers' guidelines, responses to the PAM-6 items were summed and transposed with resulting scores ranging from 0 (lowest activation) to 100 (highest activation). ${ }^{18}$

Prior analyses of the PAM have shown that patient activation develops through four stages: level 1, lack of recognition of one's role in self-care (disengaged), level 2, awareness of the need for self-care but lacking required skills and confidence (aware); level 3, actively engaged in self-care (taking action); and level 4, maintaining self-management behaviors despite challenges (maintaining behaviors). ${ }^{19}$ We used standard cut-points to categorize patients into stages of activation: level 1, PAM scores $\leq 47.0$; level 2, PAM scores 47.1-55.1; level 3, PAM scores 55.2-67.0; and level 4, PAM scores $\geq$ 67.1. ${ }^{18}$

In examining the relationship between religiosity and patient activation, we categorized patient activation levels into low (levels 1 and 2) and high (levels 3 and 4), an approach used in prior studies. ${ }^{20,21}$

\section{Baseline Covariates}

We adjusted for potential confounding by sociodemographic, psychosocial, and clinical characteristics assessed during hospitalization for ACS. Sociodemographic variables included the patient's age, sex, race/ethnicity, level of education, and marital and employment status. Healthcare barriers were measured as either a lack of usual source of care (USOC), or financial or transportation barrier.

Psychosocial measures included perceived stress, symptoms of depression and anxiety, health literacy, social support, cognitive impairment, and health-related quality of life (HRQOL). A 4-item Perceived Stress Scale (PSS4) captured the extent to which patients found their lives "uncontrollable, unpredictable, and overloading" in the prior month. ${ }^{22}$ Symptoms of depression were assessed using the 9-item Patient Health Questionnaire (PHQ-9, scores ranging from 0 to 27) with participants categorized as either having no $(\leq 4)$, mild, ${ }^{5-}$ 9 or moderate to severe $(\geq 10)$ depressive symptoms. ${ }^{23}$ Symptoms of anxiety were measured with the 7-item Generalized Anxiety Disorder (GAD-7, scores ranging from 0 to 21) Questionnaire and participants designated as having no $(\leq 4)$, mild, ${ }^{5-} 9$ or moderate to severe $(\geq 10)$ anxiety symptoms. ${ }^{24}$ From a brief screen for health literacy, participants were considered to have low health literacy if they reported having little or no confidence in filling out health forms. ${ }^{25}$ Social support was measured with 5 items from the Medical Outcomes Social Support Survey Instrument. ${ }^{26}$ Utilizing the 11-item Telephone Interview for Cognitive Status (scores ranging from 0 to 41), 
Table 1 Patient Activation Measure (6 Items)

1. When all is said and done, I am the person who is responsible for taking care of my health.

2. Taking an active role in my own healthcare is the most important thing that affects my health.

3. I am confident that I can tell whether I need to go to the doctor or whether I can take care of a health problem myself.

4. I know what treatments are available for my health problems.

5. I have been able to maintain (keep up with) lifestyle changes, like eating right or exercising.

6. I am confident that I can maintain lifestyle changes, like eating right and exercising, even during times of stress

patients with scores $\geq 33$ were classified as cognitively intact, those with scores ranging from 26 to 32 were classified as ambiguous, while those with scores $\leq 25$ were classified as having moderate to severe cognitive impairment. ${ }^{27}$ Patient's overall HRQOL was assessed using the SF-36v2 Health Survey with physical and mental well-being component summary scores ranging from 0 to $100 .^{28}$ Disease-specific HRQOL was measured with the Seattle Angina Questionnaire-QOL subscale with scores ranging from 0 to 100 , with higher scores indicating better HRQOL. ${ }^{29}$

Detailed clinical characteristics abstracted from hospital records included information on length of hospitalization, previously diagnosed comorbidities, type of ACS, and receipt of in-hospital interventional procedures including coronary artery by-pass graft (CABG) surgery and percutaneous coronary intervention (PCI).

\section{Statistical Analysis}

We compared participants who provided affirmative responses to those with non-affirmative responses to the religiosity measures according to their baseline sociodemographic, psychosocial, and clinical characteristics. Continuous variables were summarized as means and standard deviations when normally distributed and as medians and interquartile ranges when skewed. Unpaired $t$ tests, ANOVA, and the Kruskal-Wallis test were used for group comparisons of continuous variables. Chi-square tests were used to compare differences in categorical variables.

The association between religiosity and patient activation was examined both crudely and using logistic regression analysis to calculate multivariable adjusted odds ratios (aORs) and accompanying 95\% confidence intervals (CI). We included all three religiosity measures in the regression model. Multicollinearity was evaluated and ruled out by using a variance inflation factor (VIF) of $\geq 3$ to detect correlation between covariates. There was no collinearity $(\mathrm{VIF}=1.38)$ between the measures of religiosity which enhanced the development of an all-inclusive model. For multivariable adjustment, our a priori choice of potentially confounding variables was based on clinical judgment and factors known to be associated with religiosity and/or patient activation. These variables included age, sex, race/ethnicity, perceived stress, anxiety symptoms, HRQOL measures, smoking history, alcohol use, having one or more previously diagnosed comorbidities, health literacy, cognition, length of hospitalization, and in-hospital treatments received. We conducted a stratified analysis according to study site since the extent of religious involvement may have differed across our study sites in Massachusetts and Georgia.

\section{RESULTS}

Among the 2174 study participants enrolled in TRACECORE, we excluded those with missing information on the ACS subtype $(n=52)$, the PAM-6 $(n=1)$, and religiosity measures $(n=54)$, resulting in an analytic sample of 2067 patients. Study participants were on average 61.2 years old $(\mathrm{SD}=11.3), 33 \%$ were women, $81 \%$ were non-Hispanic White, and $47 \%$ had a high school degree or less. One in three patients had low health literacy, one-quarter had moderate to severe depression and anxiety, and $48 \%$ had high perceived stress. More than one-half were admitted for an NSTEMI, $80 \%$ had one or more previously diagnosed comorbidities, $52 \%$ were hospitalized for more than 3 days, and two-thirds had undergone a PCI during hospitalization.

\section{Extent of Religious Involvement}

Participants commonly reported deriving a great deal of strength and comfort from religion (52\%), one in three reported some/little strength and comfort, and 15\% indicated none. Approximately $61 \%$ reported praying for their health and $89 \%$ were aware of intercessory prayers made for their health by others.

\section{Patient Characteristics According to Religiosity}

Participants who reported deriving a great deal of strength and comfort from religion, prayed for their health, and those aware of intercessions made for their health were more likely to be women, non-Hispanic Blacks, non-users of alcohol, nonsmokers, and were less likely to be referred for cardiac rehabilitation compared with their respective counterparts who did not provide affirmative responses to each religiosity item (Table 2). Participants who endorsed all three religiosity items reported higher levels of perceived stress and lower QOL scores. Moderate to severe symptoms of depression and anxiety were more prevalent among those who prayed for their health or were aware of intercessions for their health. Participants aware of others praying for their health had greater social support (Table 2). A higher proportion of participants who derived strength and comfort from religion and those who prayed for their health were older, more likely to be cognitively impaired, had previously diagnosed comorbidities, had an NSTEMI, and underwent CABG compared with those who 


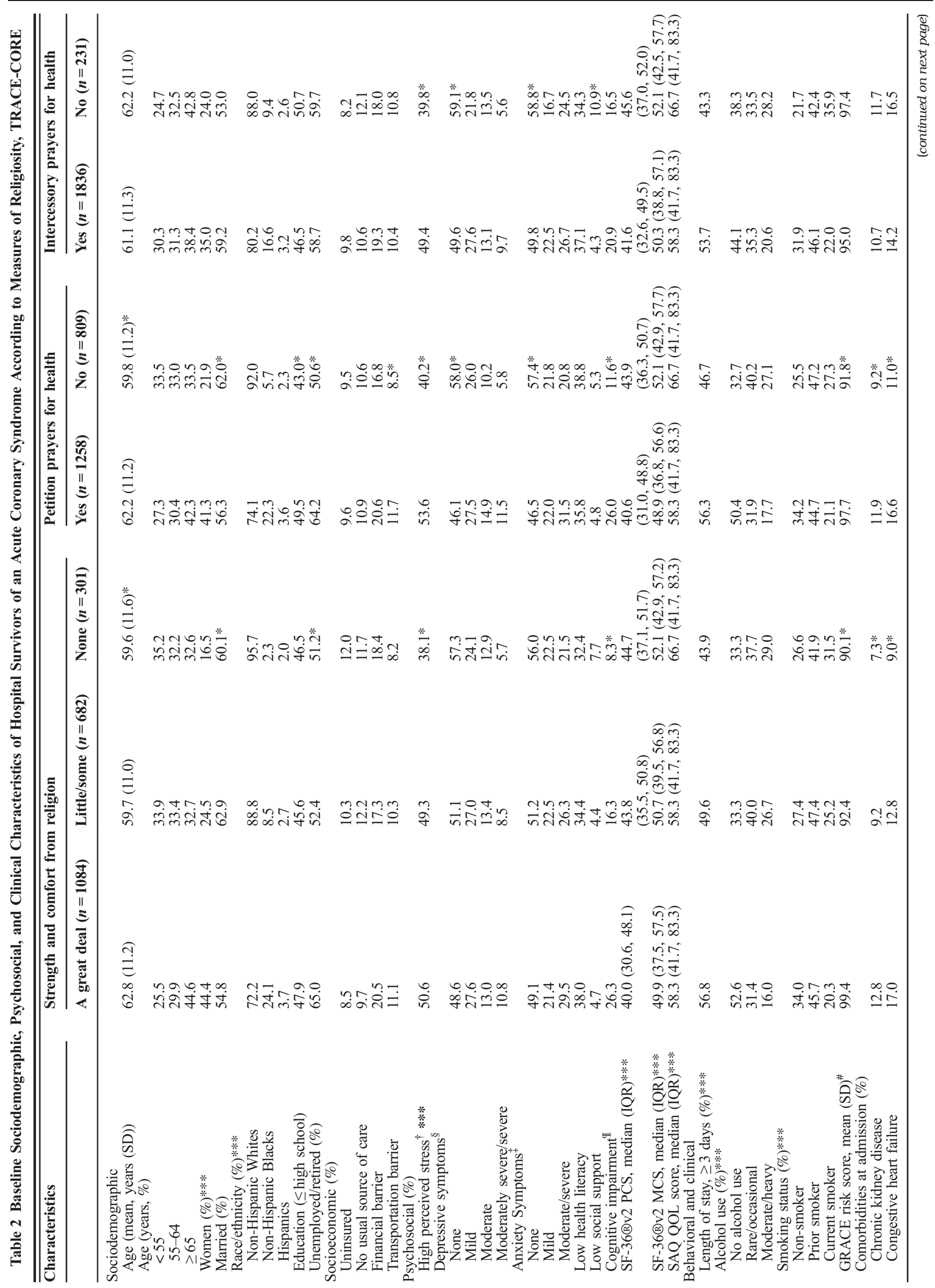




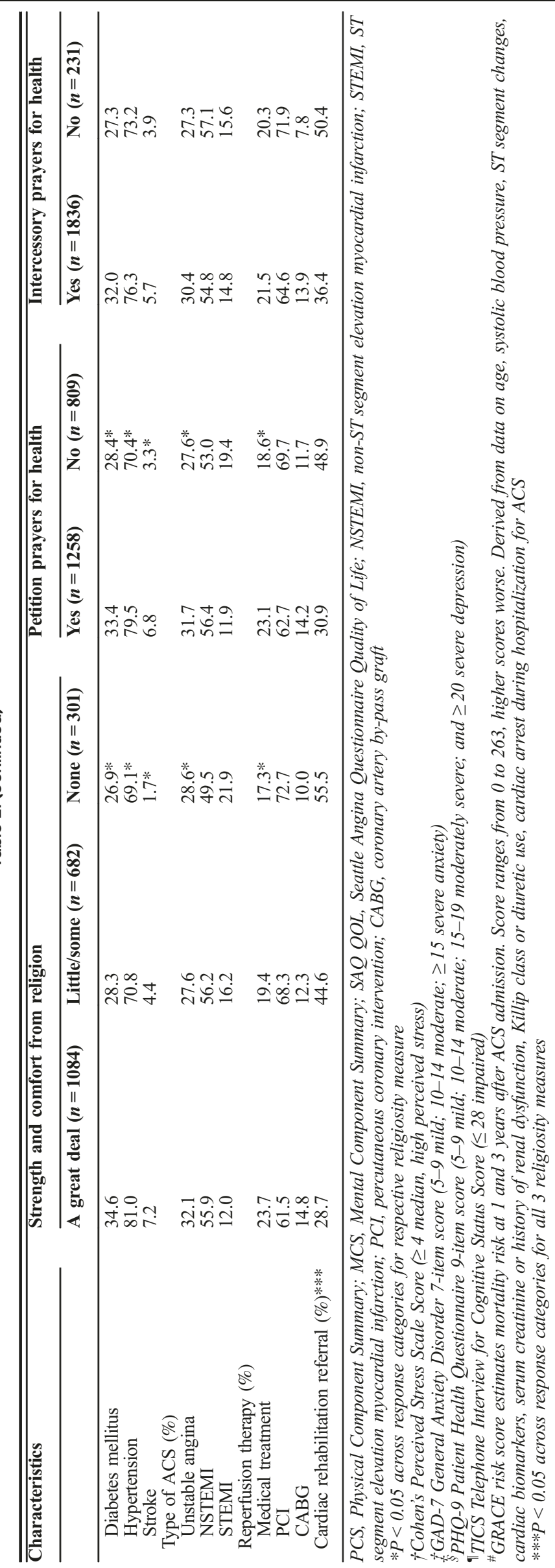


did not derive strength and comfort from religion or pray for their health $(p<0.05$ for all comparisons) (Table 2).

\section{Patient Activation During Hospitalization}

Patient activation scores were normally distributed among the study participants (mean 59.7, $\mathrm{SD}=15.2$ ). Self-reports of PAM according to the four patient activation levels were level 1, 19.9\%; level 2, 37.6\%; level 3, 20.8\%; and level 4, $21.7 \%$. Overall, $57.5 \%$ of patients had low activation (levels 1 and 2).

\section{Association Between Religiosity and Patient Activation}

After adjustment for several sociodemographic, psychosocial, and clinical characteristics, patients who reported deriving a great deal of strength and comfort from religion had higher levels of activation (aOR, 2.02; 95\% CI, 1.44-2.84), as were those who reported little/some strength and comfort (aOR, 1.45 ; 95\% CI, 1.07-1.98) compared with none (Table 3). Similarly, reports of intercessory prayers for health were associated with significantly higher activation levels (aOR, 1.48; 95\% CI, 1.07-2.05). Patients who reported praying for their health had significantly lower activation levels compared with those who did not make petition prayers for their health (aOR, 0.78; 95\% CI, 0.61-0.99) (Table 3).

Results from the stratified analyses according to study site were similar to those of the overall study findings with the exception of wider confidence intervals due to smaller sample sizes (Supplement 1).

\section{DISCUSSION}

Findings from this large observational study in a socioeconomically and racially diverse patient cohort discharged from the hospital after an ACS suggest that most acknowledge receiving strength and comfort from religion, praying for their health, and were aware of intercessory prayers made by others

Table 3 Religiosity and Patient Activation at Baseline Among Hospital Survivors of an Acute Coronary Syndrome, TRACECORE

\begin{tabular}{lll}
\hline \hline $\begin{array}{l}\text { Religiosity } \\
\text { measures }\end{array}$ & $\begin{array}{l}\text { Unadjusted OR (95\% } \\
\text { CI) }\end{array}$ & $\begin{array}{l}\text { Adjusted OR* (95\% } \\
\text { CI) }\end{array}$ \\
\hline Strength and comfort from religion & \\
A great deal & $1.65(1.20-2.26)$ & $2.02(1.44-2.84)$ \\
A little/some & $1.38(1.02-1.85)$ & $1.45(1.07-1.98)$ \\
None & Referent & Referent \\
Petition prayers for health & \\
Yes & $0.72(0.58-0.90)$ & $0.78(0.61-0.99)$ \\
No & Referent & Referent \\
Intercessory prayers for health & $1.52(1.12-2.06)$ & $1.48(1.07-2.05)$ \\
Yes & Referent & Referent \\
No &
\end{tabular}

*Adjusted for age, sex, racelethnicity, level of education, perceived stress, anxiety symptoms, physical and mental component quality of life measure, cognitive impairment, health literacy, smoking history, alcohol use, length of index hospitalization, presence of one or more comorbidities, and in-hospital treatment practices for their health. On the contrary, more than one-half of patients reported low activation levels during hospitalization. Patients who reported deriving strength and comfort from religion, and those aware of intercessions made for their health, had high activation levels, whereas patients who prayed for their health had low levels of activation.

Our findings of a high prevalence of religious engagement among hospital survivors of ACS are consistent with those of prior studies. An earlier study of 232 older patients undergoing CABG surgery found that $70 \%$ reported deriving strength and comfort from religion. ${ }^{30}$ In the Women's Health Initiative Observational Study, $87 \%$ of 92,395 participants reported receiving strength and comfort from religion. ${ }^{31}$ Furthermore, results from the Pew Research Center in 2014, which surveyed more than 35,000 Americans from all 50 states about their religious affiliations, beliefs, and practices, showed that more than half of Americans acknowledged that religion was very important in their lives and prayed daily. ${ }^{32}$

The high prevalence of religious engagement in the present and prior studies suggests that patient's religiosity could be an important social determinant of health that should be acknowledged and assessed by physicians to promote patient activation. Assessing patient's religiosity and spirituality provides a holistic approach with patient-centered management, an opportunity to understand the patient's value system, and how their religious beliefs and practices might be utilized to enhance engagement with their acute and long-term healthcare and lifestyle practices. ${ }^{33}$

The mean PAM score in the present study was 59.7 which is consistent with the average activation scores in different patient populations and settings. ${ }^{21,34}$ However, more than onehalf of our study participants had low levels of activation, in contrast with studies in patients with chronic conditions such as heart failure ${ }^{35}$ and diabetes. ${ }^{20}$ The extent of low patient activation in the present study may be explained by the sudden occurrence of their acute illness, not allowing sufficient time for engagement with their healthcare. ${ }^{35}$ Our findings highlight the need for healthcare providers to ascertain the extent of patient activation during hospitalization for ACS, and to provide clear and pertinent instructions to empower patients in successfully managing their health. Furthermore, providers should consider that the immediate period following a lifethreatening illness may be overwhelming for patients and ensure adequate provider-patient communication to address patient's health concerns.

We found that participants who acknowledged deriving strength and comfort from religion had high levels of patient activation compared with those who reported no strength and comfort. Religious beliefs and practices promote optimism, finding purpose, comfort, and meaning in suffering, and have been associated with higher QOL in varying patient populations. $^{36,37}$ These positive attitudes can foster greater levels of motivation and influence how patients adapt to life changes due to their illness, promote engagement in their healthcare, and increase patient activation. 
Research on the association between intercessory prayers and health outcomes have had equivocal findings. ${ }^{38,39} \mathrm{We}$ found that being aware of others praying for one's health was associated with high levels of patient activation. Awareness of others praying for one's health could foster a feeling of spiritual support and the recognition that others hope for one's healing, motivating the patient to adopt and maintain recommended lifestyle modifications.

Patients who prayed for their health had low levels of activation compared with those who did not pray for their health. A possible mechanism for this finding could be that patients who prayed for their health were more likely to believe in spiritual healing and were less inclined to take charge of their health. This finding supports prior studies that have shown that when individuals consider the role of God as a healer, or in extreme cases of religious fatalism in which persons believe that their health outcomes are predetermined by God and not by their actions, patients may assume a more passive role in managing their illness. ${ }^{40,41}$

A notable finding in our study is the opposite direction of the association between patient activation and petition or intercessory prayers. We acknowledge that those who prayed for their health, and those who received intercessory prayers, may differ with respect to their belief in God and the extent of their religiousness. Furthermore, those who prayed for their health may have exhibited a passive dimension of "spiritual health locus of control", making them less likely to engage in their healthcare, while those who received intercessory prayers may not have possessed such extreme religiousness that could interfere with engaging in their healthcare. Future in-depth qualitative interviews can explore the role of petition and intercessory prayers in patient engagement with their healthcare after a life-threatening illness.

Healthcare providers should increasingly be aware that religious/spiritual beliefs may influence the extent of patient activation and willingness to adopt prescribed lifestyle changes. $^{42}$ Physicians need to understand the patient's belief system and develop a mutual plan to overcome challenges that may impede patient adoption and maintenance of positive health behaviors.

\section{Study Strengths and Limitations}

We used data from a large patient cohort with sociodemographic diversity, and rich clinical, psychosocial, and behavioral information. The three measures of religiosity utilized capture how survivors of a potentially life-threatening disease incorporate religious beliefs and practices in their illness experience and recovery process. However, given our observational study design, unmeasured confounders may have potentially biased the effect estimates derived. For example, an assessment of religious denomination could have provided better understanding of how different religious regulations pertaining to health behaviors could influence patient activation. We acknowledge potential limitations in using the
PAM-6 measure to assess patient activation, since there are limited data on the use and validation of this measurement tool. Lastly, study participants were recruited from six urban hospitals in two states (Georgia and Massachusetts), and our findings may have limited generalizability to other geographic sites.

\section{CONCLUSIONS}

A majority of hospital survivors of ACS acknowledge religious practices toward their recovery. Deriving strength and comfort from religion, and receipt of intercessory prayers for health were associated with high patient activation, while petition prayers for health were associated with low activation. Our findings suggest that healthcare providers should be aware of the role of religiosity in patient engagement in their healthcare and utilize this knowledge in developing their management plans and recommendations to patients. Future research is warranted to better understand how religious beliefs and practices may influence trends in patient activation and long-term health outcomes following hospitalization for ACS.

Corresponding Author: Hawa O. Abu, MD, MPH, PhD; Department of Population and Quantitative Health Sciences University of Massachusetts Medical School, Worcester, MA, USA (e-mail: Hawa. Abu@umassmed.edu).

Funding Information Transitions, Risks, and Actions in Coronary Events: Center for Outcomes Research and Education (TRACE-CORE) was financially supported by National Institutes of Health (UO1HL105268). At the time the study was conducted, DDM was funded by the National Heart, Lung, and Blood Institute (RO1HL126911, RO1HL135219, RO1HL136660, R15HL121761). Partial support for RJG was provided by the National Heart, Lung, and Blood Institute (1R01HL126911-01A1, 5RO1HL125089-02, 5R01HL115295-05). CIK was funded by National Institutes of Health/National Institute of Mental Health (R01MH112138) and National Institutes of Health/National Center for Advancing Translational Sciences (U54 RR 026088). The funding agencies were not involved in the data collection, analysis, interpretation, writing, and submission of the paper for publication.

\section{Compliance with Ethical Standards:}

The Institutional Review Boards at participating sites approved this study. Written informed consent was obtained from each participant.

Conflict of Interest: Dr. David McManus receives sponsored research support from Bristol Myers Squibb, Pfizer, Biotronik, and Boehringer Ingelheim and has consulted for Bristol Myers Squibb, Pfizer, Samsung Electronics, and FlexCon. The other authors declare no potential conflict of interest.

\section{REFERENCES}

1. Benjamin EJ, Blaha MJ, Chiuve SE, et al. Heart Disease and Stroke Statistics-2017 Update: A Report From the American Heart Association. Circulation. 2017; 135:e146-e603.

2. Eckel RH, Jakicic JM, Ard JD, et al. 2013 AHA/ACC guideline on lifestyle management to reduce cardiovascular risk: a report of the 
American College of Cardiology/American Heart Association Task Force on Practice Guidelines. Circulation 2014; 129:S76.

3. Moljord IE, Lara-Cabrera ML, Perestelo-Pérez L, Rivero-Santana A, Eriksen L, Linaker OM. Psychometric properties of the Patient Activation Measure-13 among out-patients waiting for mental health treatment: A validation study in Norway. Patient Educ Couns 2015; 98:1410-17.

4. Hibbard $\mathbf{J H}$, Greene $\mathbf{J}$. What the evidence shows about patient activation: Better health outcomes and care experiences; fewer data on costs. Health Aff. 2013; 32: 207-14.

5. Abu HO, Anatchkova MD, Erskine NA, Lewis J, McManus DD, Kiefe CI, Santry HP. Are we "missing the big picture" in transitions of care? Perspectives of healthcare providers managing patients with unplanned hospitalization. Appl Nurs Res 2018; 44:60-66.

6. Koenig HG, Larson DB, Larson SS. Religion and coping with serious medical illness. Ann Pharmacother 2001; 35: 352-59.

7. McConnell TR, Trevino KM, Klinger TA. Demographic differences in religious coping after a first-time cardiac event. J Cardiopulm Rehabil. 2011; 31: 298-302.

8. Lucchese FA, Koenig HG. Religion, spirituality and cardiovascular disease: Research, clinical implications, and opportunities in Brazil. Rev Bras Cir Cardiovasc. 2013; 28:103-28.

9. Koenig HG. Religion, Spirituality, and Health: The Research and Clinical Implications. ISRN Psychiatry. 2012; 2012:278730.

10. Ellison CG, Levin JS. The religion-health connection: Evidence, theory, and future directions. Health Educ Behav. 1998; 25:700-720.

11. Gall TL, Charbonneau C, Clarke NH, Grant K, Joseph A, Shouldice L. Understanding the nature and role of spirituality in relation to coping and health: a conceptual framework. Can J Psychol. 2005; 46:88-104.

12. Debnam KJ, Holt CL, Clark EM, Roth DL, Foushee HR, Crowther M, Fouad M, Southward PL. Spiritual health locus of control and health behaviors in African Americans. Am J Health Behav. 2012;36(3):360-72.

13. Reyes-Ortiz CA, Rodriguez M, Markides KS. The role of spirituality healing with perceptions of the medical encounter among Latinos. J Gen Intern Med. 2009;24 Suppl 3(Suppl 3):542-547.

14. Waring ME, McManus RH, Saczynski JS, Anatchkova MD, McManus DD, Devereaux RS, Goldberg RJ, Allison JJ, Kiefe CI, TRACE-CORE Investigators. Transitions, Risks, and Actions in Coronary Events-Center for Outcomes Research and Education (TRACE-CORE): design and rationale. Circ Cardiovasc Qual Outcomes. 2012; 5:44-50.

15. Goldberg RJ, Saczynski JS, McManus DD, TRACE-CORE investigators, et al. Characteristics of Contemporary Patients Discharged From the Hospital After an Acute Coronary Syndrome. Am J Med. 2015; 128:108793

16. Anderson JL, Adams CD, Antman EM, et al. American College of Cardiology Foundation/American Heart Association Task Force on Practice Guidelines. 2012 ACCF/AHA focused update incorporated into the ACCF/AHA 2007 guidelines for the management of patients with unstable angina/non-ST-elevation myocardial infarction: a report of the American College of Cardiology Foundation/American Heart Association Task Force on Practice Guidelines. Circulation. 2013; 127:663-828.

17. Hibbard JH, Mahoney ER, Stockard J, Tusler M. Development and testing of a short form of the patient activation measure. Health Serv Res. 2005; 40:1918-30.

18. Patient Activation Measure (PAM) 6 License Materials. In: Insignia Health

19. Hibbard JH, Stockard J, Mahoney ER, Tusler M. Development of the Patient Activation Measure (PAM): conceptualizing and measuring activation in patients and consumers. Health Serv Res. 2004; 39:1005Y1026.

20. Aung E, Donald M, Williams GM, Coll JR, Doi SA. Joint influence of patient assessed chronic illness care and patient activation on glycaemic control in type 2 diabetes. Int $J$ Qual Health Care 2015; 27:117-24.

21. Zimbudzi E, Lo C, Ranasinha S, et al. Factors associated with patient activation in an Australian population with comorbid diabetes and chronic kidney disease: a cross-sectional study. BMJ Open. 2017; 7:e017695.

22. Cohen S, Williamson GM. Perceived Stress in a Probability Sample of the United States, in: S. Spacapan, S. Oskamp (Eds.), The Social Psychology of Health. Newbury Park, CA: Sage, 1988, pp. 31-67.

23. Kroenke K, Spitzer RL, Williams JBW. The PHQ-9: validity of a brief depression severity measure. J Gen Intern Med. 2001; 16:606-13.

24. Spitzer RL, Kroenke K, Williams JBW, Lowe B. A brief measure of assessing generalized anxiety disorder: the GAD-7. Arch Intern Med. 2006; 166:1092-97.

25. Powers BJ, Trinh JV, Bosworth HB. Can this patient read and understand written health information? JAMA. 2010; 304:76-84.

26. Sherbourne CD, Stewart AL. The MOS Social Support Survey. Soc Sci Med. 1991; 32:705-14

27. Ferrucci L, Lungo DI, Guralnik JM, et al. Is the telephone interview for cognitive status a valid alternative in persons who cannot be evaluated by the Mini Mental State Examination? Aging (Milano). 1998; 10:332-38.

28. Ware JEJ, Sherbourne CD. The MOS 36-item short-form health survey (SF-36). 1. Conceptual framework and item selection. Med Care. 1992; 30:473-83.

29. Spertus JA, Winder JA, Dewhurst TA, Deyo RA, Prodzinski J, McDonell M, Fihn SD. Development and evaluation of the Seattle Angina Questionnaire: a new functional status measure for coronary artery disease. JACC. 1995; 25:333-41.

30. Oxman TE, Freeman DH, Manheimer ED. Lack of social participation or religious strength and comfort as risk factors for death after cardiac surgery in the elderly. Psychosom Med. 1995; 57: 5-15.

31. Schnall E, Wassertheil-Smoller S, Swencionis C, et al. The relationship between religion and cardiovascular outcomes and all-cause mortality in the women's health initiative observational study. Psychol Health 2010; 25:249-63.

32. Pew Research Center. Religion and Public life. http://www.pewforum. org/2015/11/03/chapter-2-religious-practices-and-experiences/\#private-devotions. Accessed 4 December 2018

33. Govier I. Spiritual care in nursing: a systematic approach. Nurs Stand 2000; 14:32-6.

34. Ahn YH, Kim BJ, Ham OK, Kim SH. Factors associated with patien activation for self-management among community residents with Osteoarthritis in Korea. J Korean Acad Nurs 2015; 26:303-11.

35. Carey SA, Tecson KM, Bass K, Felius J, Hall SA. Patient activation with respect to advanced heart failure therapy in patients over age 65 years. Heart Lung. 2018; 47:285-89.

36. Koenig HG, Berk LS, Daher NS, et al. Religious involvement is associated with greater purpose, optimism, generosity and gratitude in persons with major depression and chronic medical illness. J Psychosom Res. 2014; 77: 135-43.

37. Abu HO, Ulbricht C, Ding E, Allison JJ, Salmoirgo-Blotcher E, Goldberg RJ, Kiefe CI. Association of religiosity and spirituality with quality of life in patients with cardiovascular disease: a systematic review. Qual Life Res. 2018; 27:2777-97.

38. Fosarelli P. Outcomes of Intercessory Prayer for those who are Ill: Scientific and Pastoral Perspectives. Linacre Q. 2011;78:125-37.

39. Crawford CC, Sparber AG, Jonas WB. A systematic review of the quality of research on hands-on and distance healing: Clinical and laboratory studies. Altern Ther Health Med. 2003; 9:A96-104.

40. Byrd T, Cohn LD, Gonzalez E, Parada M, Cortes M. Seatbelt use and belief in destiny among Hispanic and nonHispanic drivers. Accid Anal Prev. 1999; 31:63-5

41. Powe B, Finnie R. Cancer fatalism: the state of science. Cancer Nurs. 2003; 26:454-467.

42. Isaac KS, Hay JL, Lubetkin EI. Incorporating Spirituality in Primary Care. J Relig Health. 2016; 55: 1065-77.

Publisher's Note Springer Nature remains neutral with regard to jurisdictional claims in published maps and institutional affiliations. 